\title{
Challenges in Diagnosis and Management of Cutaneous Adverse Drug Reaction
}

\author{
Pradhan M
}

Associate Professor, Department of Dermatology, Nobel Medical College, Biratnagar, Nepal

\begin{abstract}
Adverse drug reactions commonly involve the skin and represent a significant proportion of all drug-induced illnesses. Most of the times, adverse cutaneous reactions are not severe and only a few are fatal. Real incidence of cutaneous adverse drug reaction is not known. Due to rampant and multiple drug use, there is difficulty finding the culprit drug, so diagnosis of adverse drug reaction and management becomes difficult.
\end{abstract}

Key words: Drug Eruptions; Drug Interactions; Polypharmacy

A dverse drug reactions commonly involve the skin and represent a significant proportion of all drug-induced illnesses. Most of the times, adverse cutaneous reactions are not severe and only a few are fatal. Death can result from exfoliative dermatitis, erythema multiforme or TEN. Commonly drug eruptions are reversible, abating spontaneously after the offending drug is withdrawn. ${ }^{1}$ Different morphological pattern of cutaneous adverse drug reaction has different pathophysiology and immune mediated hypersensitivity is involved in most of them.

Self-medication, polypharmacy and over the counter drug is known to contribute to Adverse Drug Reactions either by the drug itself or by causing an interaction with a prescription drug. ${ }^{2}$

For estimating the probability that a specific drug is responsible for an Adverse Drug Reaction, several scales have been developed. The most widely used is the Naranjo algorithm. ${ }^{3}$ Checking the time lapse between reaction onset and drug use, compatibility between the nature of the event and drug pharmacology, medical or pharmacological plausibility (sign and symptoms, laboratory tests and mechanism of action) are key points for a casualty assessment.

Financial disclosure: None.

Conflict of interest to disclosure: None declared.

Address of Correspondence

Dr. Manish Pradhan

Associate Professor

Department of Dermatology

Nobel Medical College, Biratnagar, Nepal.

E-mail: drmanishpradhan1@gmail.com
The most important problem in evaluating Adverse Drug Reaction is to determine the causal relationship between the drug and undesirable clinical events. It is most challenging and practically difficult when patient is on multiple drugs because of myriad clinical symptoms, poorly understood multiple mechanisms of drug-host interaction, relative paucity of laboratory testing that is available for any definitive and confirmatory drugspecific testing. Therefore, in practice, the diagnosis is purely based on clinical judgment. Comprehensive information regarding their incidence, severity and ultimate health effects are often not available as many cases go unreported so real incidence of cutaneous adverse drug reaction is not known. Due to rampant and multiple drug use, there is difficulty finding the culprit drug, so diagnosis of adverse drug reaction and management becomes difficult. This also brings difficulties in managing comorbidities. Apart from this, uneven distribution of qualified medical professional in Nepal and uncontrolled selling of drugs directly to the patients and public by retail pharmacists are the challenging issue in reducing incidence of adverse drug reaction.

Submitted: $1^{\text {st }}$ December 2018

Accepted: $3^{\text {rd }}$ February 2019

Published: $7^{\text {th }}$ October 2020

\section{How to cite this article}

Pradhan M. Challenges in diagnosis and management of cutaneous adverse drug reaction. Nepal Journal of Dermatology Venereology and Leprology 2020;18(1):1-2. https://dx.doi. org/10.3126/njdvl.v18i1.30353.

\section{(c) (7)}

Licensed under CC BY 4.0 International License which permits use, distribution and reproduction in any medium, provided the original work is properly cited. 
Pradhan $\mathrm{M}$, Challenges in diagnosis and management of cutaneous adverse drug reaction

\section{References}

1. Nayak S, Acharjya B. Adverse cutaneous drug reaction. Indian J Dermatol 2008 Jan;53(1):2. https://doi.org/10.4103/00195154.39732

2. Palaian S, Ibrahim MI, Mishra P. Pattern of adverse drug reactions reported by the community pharmacists in Nepal. Pharm Pract (Granada) 2010 Jul;8(3):201. https://doi.org/10.4321/ S1886-36552010000300008

3. Zaki S. Adverse drug reaction and causality assessment scales. Lung India $2011 \mathrm{Apr}$ 1;28(2):152.https://doi.org/10.4103/09702113.80343 